\title{
Reverse osmotic effect in active matter
}

\author{
Hyeongjoo Row $\odot$ and John F. Brady* \\ Division of Chemistry and Chemical Engineering, California Institute of Technology, Pasadena, California 91125, USA
}

(Received 18 February 2020; accepted 18 May 2020; published 11 June 2020)

\begin{abstract}
In nonequilibrium active matter systems, a spatial variation in activity can lead to a spatial variation in concentration of active particles satisfying, at steady state, the condition $n U=$ const [Schnitzer, Phys. Rev. E 48, 2553 (1993); Tailleur and Cates, Phys. Rev. Lett. 100, 218103 (2008)], where $n$ is the number density and $U$ is the active (swim) speed. We show that this condition holds even when the variation is abrupt and when thermal Brownian motion is present provided that the Péclet number is large. This spatial variation in swim speed and concentration produces a fluid pressure distribution that drives a reverse osmotic flow-fluid flows from regions of high concentration to low.
\end{abstract}

DOI: 10.1103/PhysRevE.101.062604

\section{INTRODUCTION}

Colloidal active matter systems such as motile bacteria and catalytic Janus particles display interesting out-of-equilibrium behaviors [1-13]. Moreover, these behaviors can be tuned using external means such as light $[14,15]$. Spatial control of activity can direct transport $[16,17]$, which offers a number of intriguing applications, e.g., bacteria can reproduce images by projecting light patterns $[18,19]$.

The concentration variation resulting from a spatial variation in activity was first explored by Schnitzer [20] and later by Tailleur and Cates [21-23], who showed that for slow spatial variations in one dimension the number density $n$ is inversely proportional to the speed of self-propulsion or swimming $U$, i.e., $n U=$ const. This relationship has recently been demonstrated experimentally [24].

We generalize this finding and present results for an abrupt change of activity in any dimension and include the effects of thermal Brownian motion, allowing us to span the complete range from thermal to active transport. We consider simple planar geometries that permit analytical solutions and compare the results to Brownian dynamics (BD) simulations. We show that a spatial variation in activity can be utilized as a novel pump: fluid moves from regions of high concentration to low-a reverse osmotic flow.

\section{MODELING OF MICROSWIMMERS}

The microswimmers are modeled as active Brownian particles (ABPs) that swim at speed $U$ in direction $\boldsymbol{q}$ and undergo translational and rotational Brownian motion. We limit our analysis to dilute isothermal suspensions of spherical ABPs in order to illustrate the basic physics. In addition to the swim speed, the ABPs are characterized by translational $D_{T}$ and rotational $D_{R}$ diffusivities; the latter gives the reorientation time $\tau_{R}=1 / D_{R}$. $D_{R}$ can be independent from $D_{T}=k_{B} T / \zeta$ so that athermal sources of reorientation are allowed. Here, $\zeta$ is

*jfbrady@caltech.edu the Stokes drag coefficient and $k_{B} T$ is the thermal energy. All three transport properties $U, D_{T}$, and $D_{R}$ may vary spatially. We note that since isothermal suspensions are considered, a variation in $D_{T}$ is equivalent to a spatially varying $\zeta$, which depends on the viscosity of the suspending fluid and the hydrodynamics of the low Reynolds number motion of the ABPs [25]. The behavior is governed by two intrinsic length scales [26]: the microscopic length $\delta=\sqrt{D_{T} \tau_{R}}$ and the run length $\ell=U \tau_{R}$.

The probability density $P(\boldsymbol{x}, \boldsymbol{q}, t)$ for ABPs with orientation $\boldsymbol{q}$ at position $\boldsymbol{x}$ and time $t$ is governed by the Smoluchowski equation: $\partial P / \partial t+\nabla \cdot \boldsymbol{j}_{T}+\nabla_{R} \cdot \boldsymbol{j}_{R}=\mathbf{0}$, where $\boldsymbol{j}_{T}=U \boldsymbol{q} P-D_{T} \nabla P$ is the translational flux, $\boldsymbol{j}_{R}=$ $-D_{R} \nabla_{R} P$ is the rotational flux, and $\nabla_{R}=\boldsymbol{q} \times \partial / \partial \boldsymbol{q}$ is the orientational gradient operator.

The physics is most clearly revealed by considering the first few orientational moments of the probability density; the number density $n=\int P d \boldsymbol{q}$ satisfies

$$
\frac{\partial n}{\partial t}+\nabla \cdot \boldsymbol{j}_{n}=\mathbf{0}, \quad \boldsymbol{j}_{n}=U \boldsymbol{m}-D_{T} \nabla n
$$

The polar order, $\boldsymbol{m}=\int P \boldsymbol{q} d \boldsymbol{q}$, satisfies

$$
\begin{gathered}
\frac{\partial \boldsymbol{m}}{\partial t}+\nabla \cdot \boldsymbol{j}_{\boldsymbol{m}}+(d-1) D_{R} \boldsymbol{m}=\mathbf{0} \\
\boldsymbol{j}_{\boldsymbol{m}}=U \boldsymbol{Q}+\frac{1}{d} U n \boldsymbol{I}-D_{T} \nabla \boldsymbol{m} .
\end{gathered}
$$

In (3), $\boldsymbol{Q}=\int \boldsymbol{P}(\boldsymbol{q} \boldsymbol{q}-\boldsymbol{I} / d) d \boldsymbol{q}$ is the nematic order tensor and $\boldsymbol{I}$ is the isotropic tensor. Here, $d$ is the spatial dimension for reorientation.

\section{EFFECTS OF ABRUPT VARIATIONS IN ACTIVITY}

At high activity, the Péclet number $\mathrm{Pe}=U \ell / D_{T}=$ $(\ell / \delta)^{2} \gg 1$, and the number density balance (1) for no flux is $\nabla \cdot(U \boldsymbol{m})=0$, which, for a one-dimensional (1D) geometry, requires $\boldsymbol{m}=\mathbf{0}$. From (2) at steady state the polar order is

$$
\boldsymbol{m} \approx-\frac{1}{d(d-1) D_{R}} \nabla(n U),
$$




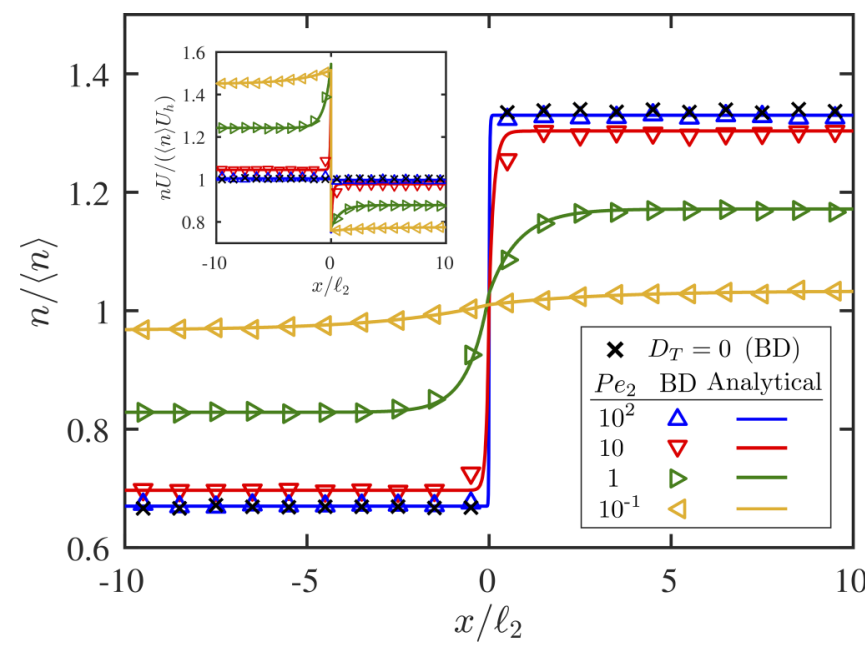

FIG. 1. Number densities scaled with $\langle n\rangle$ when $U_{1} / U_{2}=2$, $D_{T 1}=D_{T 2}$, and $D_{R 1}=D_{R 2}$ vs $x$ scaled with the run length $\ell_{2}=$ $U_{2} / D_{R 2}$. $D_{T}$ is systematically changed to show the effect of Brownian motion. Markers are BD simulations and solid lines are the analytic solutions of (1)-(3) with $\boldsymbol{Q}=\mathbf{0}$. The inset shows the product of the number density and swim speed scaled with the overall number density and the harmonic mean of the swim speeds $\left[U_{h}=2\left(U_{1}^{-1}+\right.\right.$ $\left.\left.U_{2}^{-1}\right)^{-1}\right]$.

which implies that $n U=$ const, the condition first derived for $1 D$ reorientations [20,21]. (For motion that is strictly 1D, $\boldsymbol{Q} \equiv \mathbf{0}$ because it is traceless [27].) For (4) to hold in general, $\boldsymbol{Q}$ needs to be small $(\ll n \boldsymbol{I})$ even when there is an abrupt change in properties. We show below that this is the case by a comparison to the solution of the full Smoluchowski equation. Thus, we close the hierarchy with $\boldsymbol{Q}=\mathbf{0}$ [26].

To explore the validity of $n U=$ const, when thermal Brownian motion is present and there is a sharp discontinuity in activity, we consider two cases. The first is an infinite suspension of ABPs with a step change in transport properties at $x=0$. In regions $1(x<0)$ and $2(x \geqslant 0)$, the ABPs have swim speeds $U_{i}$, translational diffusivities $D_{T i}$, and rotational diffusivities $D_{R i}$, where $i=1$ or 2 . The boundary conditions are a homogeneous random suspension far from the step change: $n_{1} \rightarrow n^{-\infty}$ and $m_{1, x} \rightarrow 0$ as $x \rightarrow-\infty$, and $n_{2} \rightarrow n^{+\infty}$ and $m_{2, x} \rightarrow 0$ as $x \rightarrow \infty$. At the discontinuity in properties, the full Smoluchowski equation requires that the field variables, $n$ and $\boldsymbol{m}$, etc., be continuous. Even though $n U=$ const would predict a discontinuity in $n$ at $x=0$, the problem is singular, and thermal Brownian motion, no matter how small (no matter how large Pe), will result in a boundary layer of thickness $O\left(\ell \mathrm{Pe}^{-1}=D_{T} / U\right)$ where Brownian motion balances advection and the probability density is continuous. Thus, $n, m_{x}$, and the fluxes $j_{n, x}$ and $j_{m, x x}$ are continuous at $x=0$. We define $\langle n\rangle$ as the scale for the number density: $\int_{-L}^{L} n d x \rightarrow 2 L\langle n\rangle$ as $L \rightarrow$ $\infty$. The moment equations are easily solved at steady state, and the full solution is provided in Appendix A. To verify the analytical solution, BD simulations have been performed as described in Appendix B.

Figure 1 shows that when activity dominates translational diffusion $(\mathrm{Pe} \gg 1$ ), the number density jumps sharply at the boundary and $n U$ becomes constant. In the absence of translational Brownian motion, indicated by the black crosses in Fig. 1, $n U$ is constant for all $x$. Increasing the diffusivities decreases Pe and smears out the sharpness of the density jump. The analytic solution gives

$$
\frac{n_{2}(+\infty)}{n_{1}(-\infty)}=\frac{C_{2} U_{1}+C_{1} U_{2}+C_{1} C_{2}^{2}\left(U_{1}-U_{2}\right)}{C_{2} U_{1}+C_{1} U_{2}-C_{1}^{2} C_{2}\left(U_{1}-U_{2}\right)},
$$

where $C_{i}=1 / \sqrt{1+d(d-1) / \mathrm{Pe}_{i}}$, showing that the number density difference is governed by the swim speed difference and modulated in amplitude by $\mathrm{Pe}_{i}=U_{i} \ell_{i} / D_{T i}$. This is in contrast to the study in [16], in which it was found via simulations that a spatial variation in number density occurred when $D_{T}$ varied even without activity. We confirmed this behavior with BD simulations and numerical solutions of the full Smoluchowski equation (see Appendix A).

Next consider a finite suspension of ABPs. The transport properties change abruptly at $x=0$, but the suspension is confined between two hard no-flux walls, $\boldsymbol{n} \cdot \boldsymbol{j}_{T}=0$, at $x=$ $-L_{1}$ and $x=L_{2}$. Continuity of field variables and fluxes at $x=0$ still applies and the overall number density is $\left(L_{1}+\right.$ $\left.L_{2}\right)\langle n\rangle=\int_{-L_{1}}^{L_{2}} n d x$. The steady-state analytical solution for bounded suspensions is straightforward to obtain, but the resulting formula is not particularly illuminating and it is difficult to evaluate because of the sharp boundary layers at both walls and at the point of discontinuity in properties. Instead, a singular perturbation analysis with three boundary layers of thickness $O\left(\ell \mathrm{Pe}^{-1}=D_{T} / U\right)$ is used along with the overall conservation of number density to obtain solutions at all positions between the walls. The steady-state analytical solution and the singular perturbation solution are provided in Appendix C.

A comparison of number densities obtained by the singular perturbation solution and BD simulations is shown in Fig. 2(a). The slight discrepancy near the walls at high Pe results from the $\boldsymbol{Q}=\mathbf{0}$ closure losing accuracy [28], not the invalidity of the singular analysis, which is valid as long as the two regions are larger than the boundary-layer thickness: $L_{1} \gg D_{T 1} / U_{1}$ and $L_{2} \gg D_{T 2} / U_{2}$.

The singular perturbation solution also accurately predicts the polar order shown in Fig. 2(b). Since $\boldsymbol{m} \sim \nabla n$ from (1) there is a peak in polar order at $x=0$ directed toward the slower region; net advective transport of particles into the slower region is balanced by the diffusive transport due to the number density jump. [Corresponding nematic order obtained by $\mathrm{BD}$ simulations is coupled to the gradient of polar order and is small $(\ll n)$ when Pe $<100$; cf. Fig. 8 in Appendix C.]

When active matter systems are confined, particles accumulate at the no-flux boundaries in sharp boundary layers [26]. The number density at a wall $n^{w}$ can be much higher than in the bulk $n^{\text {bulk }}$ [see Fig. 2(a)]. Since particles exert a local osmotic pressure $\Pi^{\text {osmo }}=n \zeta D_{T}=n k_{B} T$ at each point in space [29], the pressure exerted by active particles is higher on the wall than far from it. This extra pressure can be understood in the context of an enhanced diffusivity. Active particles undergo long-time active random walks, in addition to Brownian random walks, which increases the diffusivity of the particles by the swim diffusivity $D^{\text {swim }}=U \ell /[d(d-1)]$ [5]. Consequently, the concentration of active particles at a 

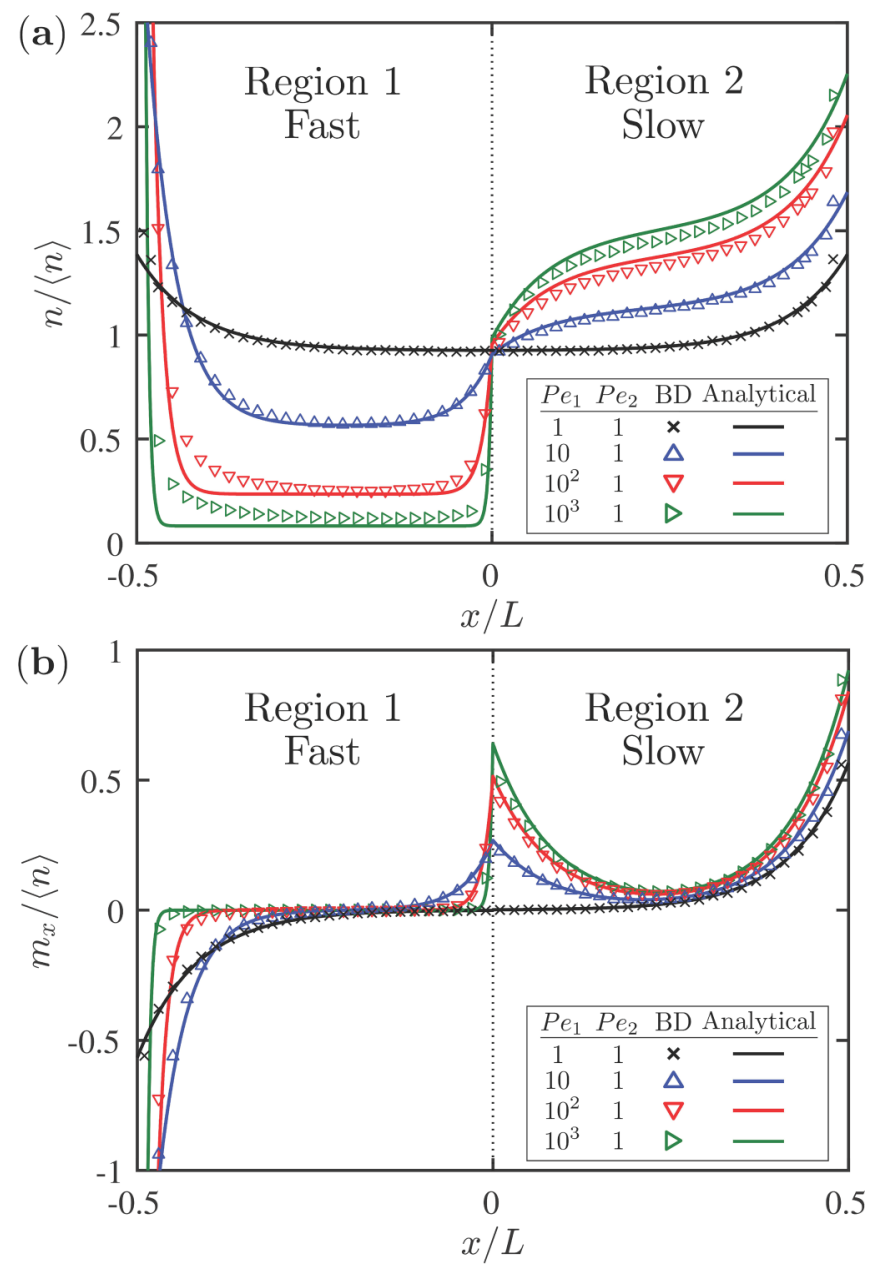

FIG. 2. (a) Number densities and (b) polar orders of confined suspensions of ABPs scaled with the overall number density $\langle n\rangle$. Markers are BD simulations and lines are analytic solutions obtained by a singular perturbation analysis. In all cases, the two regions have the same translational and rotational diffusivities and length $(L / 2)$, but the swim speeds differ: $\mathrm{Pe}_{1} / \mathrm{Pe}_{2}=\left(U_{1} / U_{2}\right)^{2}$. The coordinate $x$ is scaled with $L$, which is 10 times longer than the microscopic length scale $\delta=\sqrt{D_{T} \tau_{R}}$.

no-flux boundary is enhanced by $n^{w}=n^{\text {bulk }}\left(1+D^{\text {swim }} / D_{T}\right)$, and thus the pressure exerted on the wall is $\Pi^{w}=n^{w} k_{B} T=$ $n^{\text {bulk }} \zeta\left(D_{T}+D^{\text {swim }}\right)$; the contribution $n^{\text {bulk }} \zeta D^{\text {swim }}$ is the swim pressure $[5,26]$. Since $n^{\text {bulk }} U$ is nearly constant for highly active particles $\left(\mathrm{Pe} \approx D^{\mathrm{swim}} / D_{T} \gg 1\right)$, the pressure exerted by particles on the walls $\Pi^{w} \approx n^{\text {bulk }} \zeta D^{\text {swim }} \sim \zeta\left(n^{\text {bulk }} U\right) \ell$ will be different in the two regions if the run lengths are different $\ell_{1} \neq \ell_{2}$.

To test this prediction, we directly measured the pressure exerted by particles on the walls $\Pi^{w}$. From the analytical solution, the pressure is computed from the number density at the wall, while for BD simulations the force transmitted when each APB collides with a wall is monitored. As shown in Fig. 3, the ratio of pressures exerted by ABPs on the two walls is predicted very accurately. At large Pe the pressure is predicted to be linear in the run length, which is borne out precisely as shown in Fig. 3.

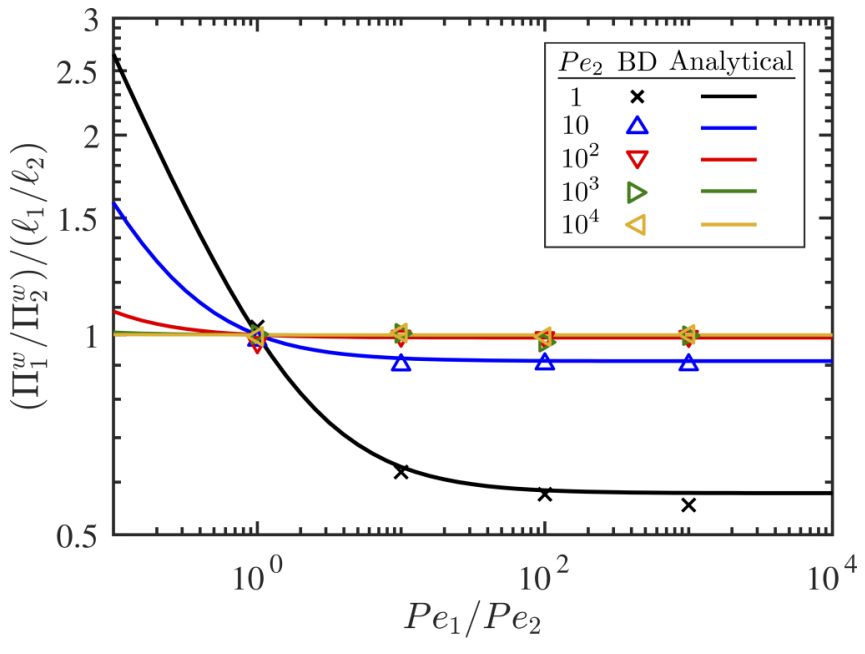

FIG. 3. The ratio of the particle pressures exerted by ABPs on wall $1\left(z=-L_{1}\right)$ and wall $2\left(z=L_{2}\right)$ scaled by the ratio of corresponding run lengths. Markers are BD simulations and lines are the singular perturbation solutions. The two regions have the same length and transport properties except for the swim speed $\left[\mathrm{Pe}_{1} / \mathrm{Pe}_{2}=\left(U_{1} / U_{2}\right)^{2}\right]$. The distance between the walls $L=L_{1}+L_{2}$ is 100 times longer than the microscopic length scale $\delta_{2}$ in region 2 . In the high activity limit $(\mathrm{Pe} \rightarrow \infty)$, the ratio of pressures exerted by particles on the walls is $\ell_{1} / \ell_{2}$.

\section{FLUID PRESSURE DISTRIBUTION AND REVERSE OSMOTIC EFFECT}

The difference in pressure exerted by ABPs on the walls must be balanced by a difference in pressure in the suspending fluid, otherwise there would be a net force exerted on the container. Active motion is a force-free motion-particles push off the suspending fluid and their motion is balanced by the fluid drag. A container of active matter cannot exert any net force on its boundary. This is clear for so-called "wet" active matter-particles immersed in a fluid-but it also applies to "dry" active matter such as crawling particles that push off a substrate-the combination of particles and substrate is force-free.

The force-free condition requires the total pressure in the suspension to be constant (when there is no flow). The total pressure $P$ is the sum of the local osmotic pressure $\Pi^{\text {osmo }}=$ $n k_{B} T$ exerted by particles and the fluid pressure $p_{f}$. Since $P=$ $p_{f}+n k_{B} T=$ const, the fluid pressure distribution is opposite that of the number density. From (1), the gradient of the fluid pressure is directly related to the polar order and the flux of the active particles:

$$
\nabla p_{f}=-\zeta U \boldsymbol{m}+\zeta \boldsymbol{j}_{n}
$$

Equation (6) is a momentum balance on the fluid. The right-hand side is the net hydrodynamic force exerted by active particles on the fluid [30]. The total hydrodynamic force on an individual swimmer $\boldsymbol{F}_{\alpha}^{\text {hydro }}=-\zeta\left(\boldsymbol{U}_{\alpha}-\boldsymbol{u}_{f}\right)+\zeta U \boldsymbol{q}_{\alpha}$ consists of the propulsive swim force $\boldsymbol{F}_{\alpha}^{\text {swim }}=\zeta U \boldsymbol{q}_{\alpha}$ and the fluid drag $\boldsymbol{F}_{\alpha}^{\text {drag }}=-\zeta\left(\boldsymbol{U}_{\alpha}-\boldsymbol{u}_{f}\right)$. Here, $\alpha$ is the index of each swimmer. Averaging over a continuum volume element with number density $n$ then gives $n\left\langle\boldsymbol{F}^{\text {hydro }}\right\rangle=-\zeta \boldsymbol{j}_{n}+\zeta U \boldsymbol{m}$. The particle flux is $\boldsymbol{j}_{n}=n\left(\boldsymbol{u}_{p}-\boldsymbol{u}_{f}\right)$, where $\boldsymbol{u}_{p}=\frac{1}{N} \sum_{\alpha} \boldsymbol{U}_{\alpha}$ is the 
average particle velocity. The drag force is proportional to the velocity of a particle relative to the fluid, $\boldsymbol{U}_{\alpha}-\boldsymbol{u}_{f}$, where $\boldsymbol{u}_{f}$ is the fluid velocity. Only relative motion produces a drag force, a requirement of Galilean invariance.

The fluid momentum balance (6) shows that if there is net polar order - a net swim force-in any region this can be balanced by a net particle flux, $\boldsymbol{j}_{n}=U \boldsymbol{m}$, with $\nabla p_{f}=0$, or if there is no particle flux, $\boldsymbol{j}_{n}=0$, then there must be a fluid pressure gradient, $\nabla p_{f} \neq 0$.

How can we understand the origin of this fluid pressure gradient? When an active particle moves, an equal volume of fluid is displaced in the opposite direction - there is no net mass (or volume) flux for force-free motion. (For "dry" active matter, when one takes a step an equivalent amount of ground "moves" in the opposite direction.) Out in the bulk where particles are swimming randomly and there is no polar order, there is no net flux of material across any plane. At a no-flux boundary the active particle's velocity normal to the boundary is zero; the force from the boundary balances the swim force. However, the particle has not stopped its "swim strokes" and thus it is still displacing fluid away from the boundary. Since the fluid does not exit (or enter) the boundary, there must be a fluid pressure difference between the wall and the bulk to shut off the flow generated by the active swimming.

This is easily seen from the solution for the concentration and polar order adjacent to a plane wall. From [26], $m_{z}=-\frac{1}{6} n^{\text {bulk }} \ell \lambda e^{-\lambda z}$, where the accumulation boundary layer thickness $\lambda^{-1}=\delta / \sqrt{2\left(1+D^{\text {swim }} / D_{T}\right)}$. From (6), $\partial p_{f} / \partial z=-\zeta U m_{z}=n^{\text {bulk }} \zeta U \ell \lambda e^{-\lambda z} / 6$ and thus $p_{f}^{w}-$ $p_{f}^{\text {bulk }}=-n^{\text {bulk }} \zeta D^{\text {swim }}$. The drop in fluid pressure at the wall is precisely equal to the swim pressure exerted by the active particles on the boundary. The total pressure $P=p_{f}^{w}+n^{w} k_{B} T=$ $p_{f}^{\text {bulk }}+n^{\text {bulk }} k_{B} T$ is constant at each location and there is no net flux of particles or fluid in the system.

A fluid pressure jump occurs not just adjacent to noflux walls, but also at a point of discontinuity in the swim speed. As seen in Fig. 2(b), there is net polar order across the interface pointing into the slower region and a jump in concentration and thus a jump in the osmotic pressure. Since the overall pressure is constant, the jump in fluid pressure $\Delta p_{f}=p_{f 1}^{\text {bulk }}-p_{f 2}^{\text {bulk }}=\left(n_{2}^{\text {bulk }}-n_{1}^{\text {bulk }}\right) k_{B} T=$ $\left(n_{1}^{\text {bulk }}+n_{2}^{\text {bulk }}\right) k_{B} T\left(U_{1}-U_{2}\right) /\left(U_{1}+U_{2}\right)$, where we have made use of $n U=$ const. A fluid pressure difference arises from a difference in swim speeds.

Now suppose that instead of an impermeable wall, the no-flux boundaries are semipermeable membranes allowing passage of fluid but not particles. Active particles accumulated at the membranes can act_-via their swim strokes—as a pump for the fluid. This pumping action is illustrated in Fig. 4. Because the run length in the fast region is larger than in the slow region, the concentration at the fast boundary exceeds that at the slow boundary, $n_{1}^{w}>n_{2}^{w}$, even though the bulk concentration is lower, $n_{1}^{\text {bulk }}<n_{2}^{\text {bulk }}$. Thus, the fluid pressure at the fast wall is less than at the slow wall, $p_{f 1}^{w}<p_{f 2}^{w}$, and fluid will be sucked into the container at the fast wall and ejected at the slow wall. From the perspective of the bulk concentration this appears to be a reverse osmotic effectfluid is sucked into the region with the lower concentration.

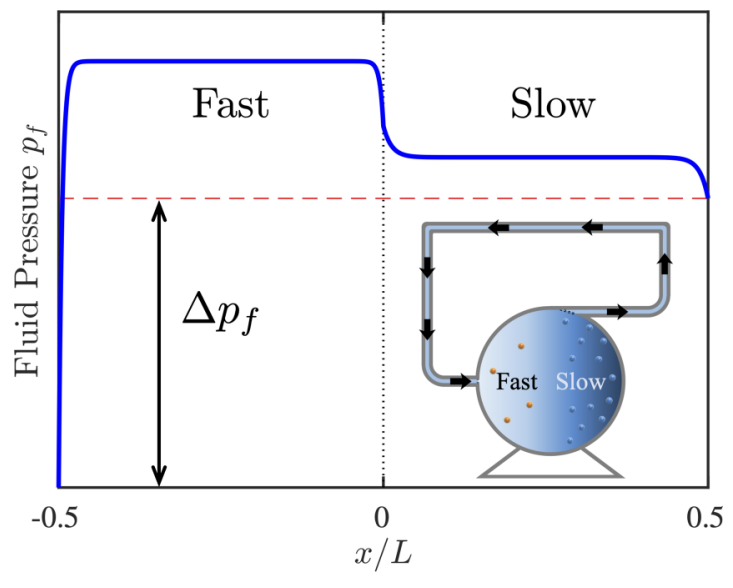

FIG. 4. A schematic of the fluid pressure distribution and a novel pumping device powered by the activity of suspended particles. Since the pressure exerted on a wall by active particles is larger in the region with the faster swim speed, the fluid pressure is smaller there. Thus, when two regions with different swim speeds are connected by a tube and the walls are semipermeable membranes, fluid will flow from the slower to the faster region-from regions of high concentration to low.

The speed of the pumped fluid can be estimated as follows (see Appendix D for details). A suspension of active particles of $1 \mu \mathrm{m}$ radius, reorientation time $10 \mathrm{~s}$, and volume fraction $\phi=0.2$, with swim speeds 5 and $10 \mu \mathrm{m} / \mathrm{s}$ in two regions, produces a flow in a cylindrical-shaped connecting tube of radius $1 \mathrm{~cm}$ of approximately $0.1 \mu \mathrm{m} / \mathrm{s}$ (when the semipermeable membranes are $100 \mathrm{~cm}^{2}$ wide with a resistance of $10^{9} \mathrm{~m}^{-1}$ ). Here, we assumed that the flow does not significantly disturb the concentration or pressure distributions within the bulk regions, which is true when $\mathrm{Pe}_{U}=$ $u_{s} L / D^{\text {swim }} \ll 1$, where $u_{s}$ is the flow speed in the suspension container (not the tube) and $L$ is the size of the container. If $\mathrm{Pe}_{U}$ is not small, one needs to solve the Smoluchowski equation including the advective flux $\boldsymbol{u}_{S} P$ in the translational flux $\boldsymbol{j}_{T}$.

We have considered dilute suspensions in this paper, but the physical processes associated with a variation in transport properties should be qualitatively the same even when particle-particle interactions are significant. To determine fluid pressure distributions in such systems, the mechanical balance still applies with the inclusion of the collisional pressure of particles [5].

\section{ACKNOWLEDGMENT}

This research was supported in part by NSF under Grant No. 1803662.

\section{APPENDIX A: ANALYTICAL AND NUMERICAL SOLUTIONS FOR AN INFINITE SUSPENSION OF ACTIVE BROWNIAN PARTICLES}

We present an analytic solution for the infinite suspension of active Brownian particles (ABPs) with a step change in transport properties obtained with the $\boldsymbol{Q}=\mathbf{0}$ closure. In regions $1(x<0)$ and $2(x \geqslant 0)$ the ABPs have swim speeds 
$U_{i}$, translational diffusivities $D_{T i}$, and rotational diffusivities $D_{R i}$, where $i=1$ or 2 . The overall number density is $\langle n\rangle=$ $\lim _{L \rightarrow \infty} \int_{-L}^{L} n d x /(2 L)$. By solving (1)-(3), we obtain

$$
\begin{gathered}
m_{i, x}=m_{x 0} e^{ \pm \lambda_{i} x}, \\
\frac{n_{i}}{n^{\mp \infty}}=1+\frac{C_{i}\left(U_{1}-U_{2}\right)}{ \pm\left(\frac{U_{1}}{C_{1}}+\frac{U_{2}}{C_{2}}\right)-C_{i}\left(U_{1}-U_{2}\right)} e^{ \pm \lambda_{i} x},
\end{gathered}
$$

where

$$
\begin{gathered}
\frac{m_{x 0}}{\langle n\rangle}=\frac{\frac{1}{\sqrt{d}}\left(U_{1}-U_{2}\right)}{\left(\frac{U_{1}}{C_{1}}+\frac{U_{2}}{C_{2}}\right)-\frac{1}{2}\left(U_{1}-U_{2}\right)\left(C_{1}-C_{2}\right)}, \\
\frac{n^{\mp \infty}}{\langle n\rangle}=\frac{\left(\frac{U_{1}}{C_{1}}+\frac{U_{2}}{C_{2}}\right) \mp C_{i}\left(U_{1}-U_{2}\right)}{\left(\frac{U_{1}}{C_{1}}+\frac{U_{2}}{C_{2}}\right)-\frac{1}{2}\left(U_{1}-U_{2}\right)\left(C_{1}-C_{2}\right)}, \\
\lambda_{i}=\frac{\sqrt{d-1}}{\delta_{i}} \sqrt{1+\frac{\mathrm{Pe}_{i}}{d(d-1)}}, \\
C_{i}=\frac{1}{\sqrt{1+\frac{d(d-1)}{\mathrm{Pe}_{i}}}} .
\end{gathered}
$$

Here, the subscript $i$ indicates the two regions (1 or 2), $\delta_{i}=$ $\sqrt{\overline{D_{T i} / D_{R i}}}$ is the microscopic length scale related to the translational diffusion, $\mathrm{Pe}_{i}=U_{i} \ell_{i} / D_{T i}=U_{i}^{2} /\left(D_{T i} D_{R i}\right)=\left(\ell_{i} / \delta_{i}\right)^{2}$ is the Péclet number, which is a measure of the activity of the ABPs, $n^{\mp \infty}$ is the number density far from the step change in transport properties $(x \rightarrow \mp \infty), d$ is the spatial dimension for reorientation, upper signs are for region $1(x<0)$, and lower signs are for region $2(x \geqslant 0)$. Note that the inverse screening length $\lambda_{i}$ is the characteristic length scale of the decay of step changes in the field variables. A modified Péclet number $C_{i}$ ranges from 0 when $\mathrm{Pe}_{i}=0$ to 1 when $\mathrm{Pe}_{i} \rightarrow \infty$. The overall number density is simply $\langle n\rangle=\left(n^{-\infty}+n^{+\infty}\right) / 2$ for an infinite suspension.

The analytical solution predicts that the number density and polar order are governed by the swim speed and modulated in amplitude by the product of the translational and rotational diffusivities $D_{T} D_{R}$. When there is no difference in swim speeds $\left(U_{1}-U_{2}=0\right)$, the suspension is homogeneous; the number density is uniform and the polar order is zero everywhere. Also, the number density is always lower in the region with higher swim speed, and the nonzero polar order points toward the region with slower swim speed. A typical distribution of number densities $n^{+\infty} / n^{-\infty}$ is presented in Fig. 5.

The number density changes rapidly only near $x=0$. Since $\boldsymbol{m} \sim \nabla n$, the polar order field is nearly zero everywhere except near $x=0$, where it has a peak as seen in Fig. 6(b). The sharpness of the peak increases as the screening length $\lambda^{-1}$ decreases. In the singular athermal limit $\left(D_{T} \rightarrow 0\right)$, the number density satisfies $n U=$ const and the polar order becomes a $\delta$-function if swim speeds are different in the two regions.

We have obtained numerical solutions of the full Smoluchowski equation with the finite-element method in order to verify the predictions of the analytical solution (A1)-(A6) obtained by the $\boldsymbol{Q}=\mathbf{0}$ closure. Figure 6 shows that solutions of the full Smoluchowski equation agree with the $\boldsymbol{Q}=\mathbf{0}$

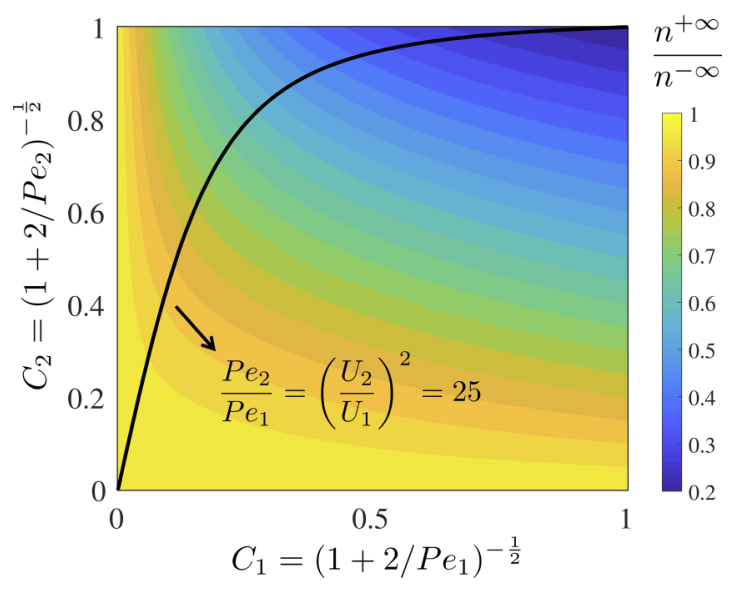

FIG. 5. Contour plot of $n^{+\infty} / n^{-\infty}$ for $U_{2} / U_{1}=5$. The modified Péclet numbers $C_{i}=0$ when $\mathrm{Pe}_{i}=0$ and $C_{i} \rightarrow 1$ when $\mathrm{Pe}_{i} \rightarrow \infty$. The black line represents the case when diffusivities are the same in the two regions. When activities in both regions are high $\left(C_{1}, C_{2} \approx\right.$ 1), $n^{+\infty} / n^{-\infty} \approx U_{1} / U_{2}=0.2$, i.e., $n U=$ const. If either of the two regions is diffusion-dominated $\left(C_{i} \approx 0\right)$, the number density becomes constant throughout the whole suspension: $n^{+\infty}=n^{-\infty}$.

closure and that the variation in the number density and the peak of the polar order only occur when the swim speed is different in the two regions.

If suspended particles are not active in one region, say region 2 (i.e., $U_{2}=0$ ), the analytical solution is given by

$$
\begin{gathered}
m_{1, x}=m_{x 0} e^{\lambda_{1} x}, \\
m_{2, x}=m_{x 0} e^{\frac{x}{\delta_{2}}}, \\
\frac{n_{1}}{n^{-\infty}}=1+\frac{C_{1} \mathrm{Pe}_{1,2}^{\frac{1}{2}} e^{\lambda_{1} x}}{\left(\frac{1}{C_{1}}-C_{1}\right) \mathrm{Pe}_{1,2}^{\frac{1}{2}}+1}, \\
n_{2}=n^{+\infty},
\end{gathered}
$$

where

$$
\begin{aligned}
\frac{m_{x 0}}{\langle n\rangle} & =\frac{\frac{1}{\sqrt{d}} \mathrm{Pe}_{1,2}^{\frac{1}{2}}}{\left(\frac{1}{C_{1}}-\frac{C_{1}}{2}\right) \mathrm{Pe}_{1,2}^{\frac{1}{2}}+1}, \\
\frac{n^{-\infty}}{\langle n\rangle} & =\frac{\left(\frac{1}{C_{1}}-C_{1}\right) \mathrm{Pe}_{1,2}^{\frac{1}{2}}+1}{\left(\frac{1}{C_{1}}-\frac{C_{1}}{2}\right) \mathrm{Pe}_{1,2}^{\frac{1}{2}}+1}, \\
\frac{n^{+\infty}}{\langle n\rangle} & =\frac{\frac{1}{C_{1}} \mathrm{Pe}_{1,2}^{\frac{1}{2}}+1}{\left(\frac{1}{C_{1}}-\frac{C_{1}}{2}\right) \mathrm{Pe}_{1,2}^{\frac{1}{2}}+1}, \\
\mathrm{Pe}_{1,2} & =\frac{U_{1}^{2}}{d(d-1) D_{T 2} D_{R 2}} .
\end{aligned}
$$

Here, $\mathrm{Pe}_{1,2}$ is the global Péclet number and it represents the relative significance of advective transport in region 1 compared to diffusive transport in region 2.

By comparing (A1)-(A6) and (A7)-(A14), we observe an interesting difference between passive-like ABPs $(U>0$ and $\mathrm{Pe} \rightarrow 0$ ) and passive Brownian particles (PBPs; $U=0$ and so $\mathrm{Pe}=0$ ), which show the identical diffusion-dominated dynamics [31,32]. 
(a)

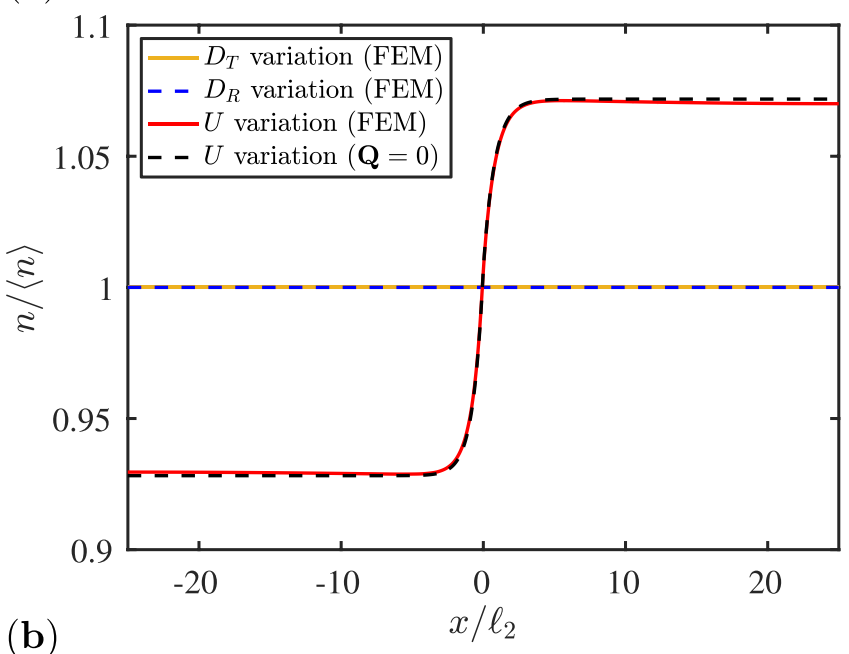

(b)

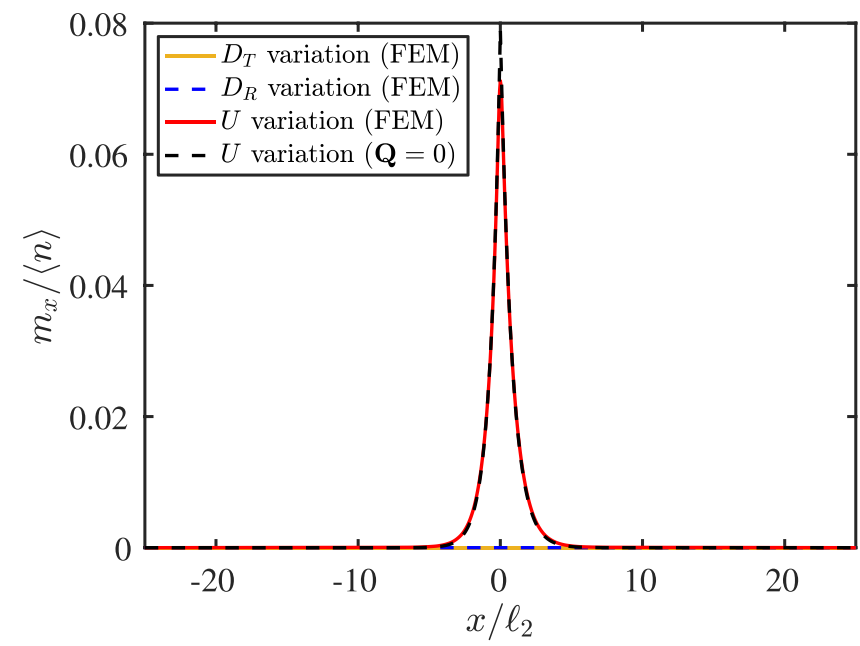

FIG. 6. (a) Number densities and (b) polar orders scaled with $\langle n\rangle$. Black dashed line represents the analytical solution obtained by the $\boldsymbol{Q}=\mathbf{0}$ closure [(A1)-(A6)] and the other lines are obtained by solving the full Smoluchowski equation with the finite-element method. The position $x$ is scaled with the run length $\ell_{2}$ in region 2. In region $1(x<0), \mathrm{Pe}=2$ and in region $2(x \geqslant 0), \mathrm{Pe}=1$. The number density changes only when there exists variation of swim speed. Also, the assumption of $\boldsymbol{Q}=\mathbf{0}$ does not compromise the accuracy significantly when $\mathrm{Pe}<100$ for which the effect of a nematic field is not prominent.

\section{APPENDIX B: BROWNIAN DYNAMICS SIMULATIONS}

The Brownian dynamics (BD) simulation is a numerical solution of the overdamped Langevin equations, which describe the motion of an ABP:

$$
\begin{gathered}
0=-\zeta \dot{\boldsymbol{x}}+\boldsymbol{F}^{\mathrm{swim}}+\boldsymbol{F}^{B}, \\
0=-\zeta_{R} \boldsymbol{\Omega}+\boldsymbol{L}^{R},
\end{gathered}
$$

where $\dot{\boldsymbol{x}}$ is translational velocity of the particle, $\boldsymbol{\Omega}$ is its angular velocity, $\zeta$ is the translational drag coefficient, $\zeta_{R}$ is the rotational drag coefficient, $\boldsymbol{F}^{\text {swim }}$ is the swim force for self-propulsion, $\boldsymbol{F}^{B}$ is random Brownian force, and $\boldsymbol{L}^{R}$ is torque for reorientation of the particle.
The swim force $\boldsymbol{F}^{\text {swim }}=\zeta \boldsymbol{U} \boldsymbol{q}$ is exerted toward the swimming direction of a particle $\boldsymbol{q}(\boldsymbol{q} \cdot \boldsymbol{q}=1)$ with a magnitude of $\zeta U$, where $U$ is the swim speed of an ABP. The random Brownian force $\boldsymbol{F}^{B}$ is defined with conventional white noise properties: $\overline{\boldsymbol{F}^{B}(t)}=0$ and $\overline{\boldsymbol{F}^{B}(0) \boldsymbol{F}^{B}(t)}=2 k_{B} T \zeta \delta(t) \boldsymbol{I}$, where $\tau$ is the ensemble average. Similarly, the reorientation torque $\boldsymbol{L}^{R}$ is defined as $\overline{\boldsymbol{L}^{R}(t)}=0$ and $\overline{\boldsymbol{L}^{R}(0) \boldsymbol{L}^{R}(t)}=2 \zeta_{R}^{2} \delta(t) \boldsymbol{I} / \tau_{R}$. The swimming direction of a particle changes due to its angular speed: $d \boldsymbol{q} / d t=\boldsymbol{\Omega} \times \boldsymbol{q}$.

The $\mathrm{BD}$ simulation is performed by integrating the Langevin equations numerically in time. We have used the explicit Euler method with intrinsic error from the numerical method of $O\left((\Delta t)^{2}\right)$. However, errors of $O(\Delta t)$ occur in positions of particles whenever they pass the interface of the two regions. Therefore, the overall magnitude of error of simulations is $O(\Delta t)$. Our choice of $\Delta t$ ensures that errors in positions of particles from each step are at most an order of $0.1 \%$ of the shortest length scale in the system.

For interactions between particles and walls, we use the potential-free algorithm [33] to model the infinite force from hard walls. For the 2D simulations presented, the pressure exerted by particles on a wall is obtained by dividing the force exerted by particles on the wall with the length of the wall.

\section{APPENDIX C: ANALYTICAL SOLUTIONS TO FINITE SUSPENSIONS OF ACTIVE BROWNIAN PARTICLES}

We now consider a suspension of ABPs between two hard walls when there is a step change in transport properties in

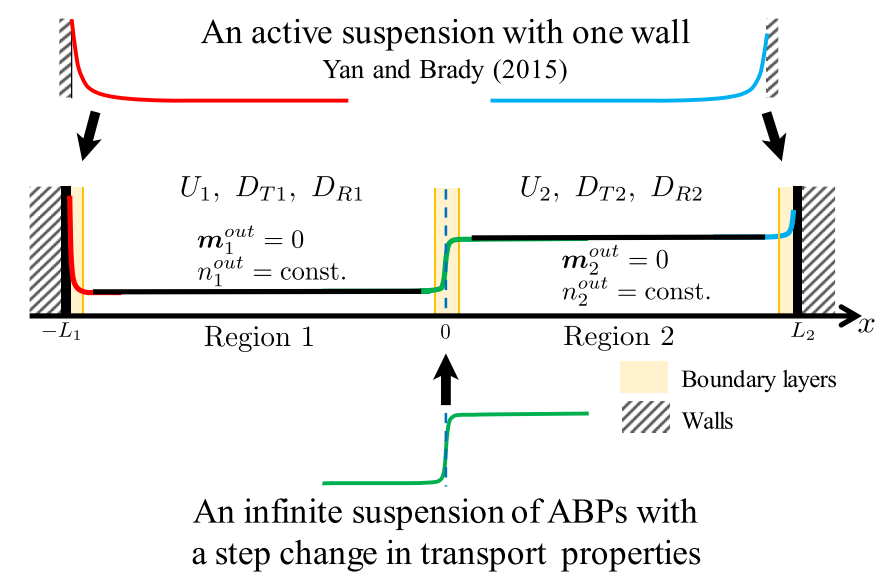

FIG. 7. A schematic of the singular perturbation analysis with matched asymptotic expansions. Red and blue lines represent the number density in boundary layers near walls. If the macroscopic length scale is significantly larger than the thickness of the boundary layers, we can approximate the suspension as if it is semi-infinite with a wall. The leading order solution has been obtained by Yan and Brady [26]. The green line represents the number density in the boundary layer where transport properties change. Similarly, a sufficiently large suspension can be approximated as an infinite suspension with a step change in transport properties, which we discussed above. The number densities in boundary layers are matched with number densities in bulk or outer regions where the number density is constant to leading order. 
the region between the two walls as illustrated in Fig. 7. The step change occurs at $x=0$ and the two walls are located at $x=-L_{1}(<0)$ and $x=L_{2}(>0)$, respectively. Again, in regions $1\left(-L_{1} \leqslant x<0\right)$ and $2\left(0 \leqslant x \leqslant L_{2}\right)$ the ABPs have swim speeds $U_{i}$, translational diffusivities $D_{T i}$, and rotational diffusivities $D_{R i}$, where $i=1$ or 2 .

An analytical solution with the $\boldsymbol{Q}=\mathbf{0}$ closure is obtained by solving (1)-(3) as

$$
\begin{aligned}
& \frac{m_{i, x}}{n_{0}}=\frac{m_{x 0}}{n_{0}} \cosh \left(\lambda_{i} x\right)+a_{i} \sinh \left(\lambda_{i} x\right), \\
\frac{n_{i}}{n_{0}}= & \gamma_{i} a_{i}\left[\cosh \left(\lambda_{i} x\right)-1\right]+\gamma_{i} \frac{m_{x 0}}{n_{0}} \sinh \left(\lambda_{i} x\right)+1,
\end{aligned}
$$

where

$$
\begin{aligned}
& \frac{m_{x 0}}{n_{0}}=\frac{\Lambda_{1} \Lambda_{2}\left(U_{1}-U_{2}\right)\left[\cosh \left(\lambda_{1} L_{1}\right)-1\right]\left[\cosh \left(\lambda_{2} L_{2}\right)-1\right]+\Lambda_{1} U_{1}\left[\cosh \left(\lambda_{1} L_{1}\right)-1\right]-\Lambda_{2} U_{2}\left[\cosh \left(\lambda_{2} L_{2}\right)-1\right]}{\frac{d}{\gamma_{1}} \Lambda_{1} U_{1} \sinh \left(\lambda_{1} L_{1}\right)\left\{1+\Lambda_{2}\left[\cosh \left(\lambda_{2} L_{2}\right)-1\right]\right\}+\frac{d}{\gamma_{2}} \Lambda_{2} U_{2} \sinh \left(\lambda_{2} L_{2}\right)\left\{1+\Lambda_{1}\left[\cosh \left(\lambda_{1} L_{1}\right)-1\right]\right\}}, \\
& a_{1}=\frac{\Lambda_{1} \Lambda_{2}\left(U_{1}-U_{2}\right) \sinh \left(\lambda_{1} L_{1}\right)\left[\cosh \left(\lambda_{2} L_{2}\right)-1\right]+\Lambda_{1} U_{1} \sinh \left(\lambda_{1} L_{1}\right)+\frac{\gamma_{1}}{\gamma_{2}} \Lambda_{2} U_{2} \sinh \left(\lambda_{2} L_{2}\right)}{\frac{d}{\gamma_{1}} \Lambda_{1} U_{1} \sinh \left(\lambda_{1} L_{1}\right)\left\{1+\Lambda_{2}\left[\cosh \left(\lambda_{2} L_{2}\right)-1\right]\right\}+\frac{d}{\gamma_{2}} \Lambda_{2} U_{2} \sinh \left(\lambda_{2} L_{2}\right)\left\{1+\Lambda_{1}\left[\cosh \left(\lambda_{1} L_{1}\right)-1\right]\right\}}, \\
& a_{2}=\frac{\Lambda_{1} \Lambda_{2}\left(U_{2}-U_{1}\right) \sinh \left(\lambda_{2} L_{2}\right)\left[\cosh \left(\lambda_{1} L_{1}\right)-1\right]+\frac{\gamma_{2}}{\gamma_{1}} \Lambda_{1} U_{1} \sinh \left(\lambda_{1} L_{1}\right)+\Lambda_{2} U_{2} \sinh \left(\lambda_{2} L_{2}\right)}{\frac{d}{\gamma_{1}} \Lambda_{1} U_{1} \sinh \left(\lambda_{1} L_{1}\right)\left\{1+\Lambda_{2}\left[\cosh \left(\lambda_{2} L_{2}\right)-1\right]\right\}+\frac{d}{\gamma_{2}} \Lambda_{2} U_{2} \sinh \left(\lambda_{2} L_{2}\right)\left\{1+\Lambda_{1}\left[\cosh \left(\lambda_{1} L_{1}\right)-1\right]\right\}}, \\
& \lambda_{i}=\frac{\sqrt{d-1}}{\delta_{i}} \sqrt{1+\frac{\mathrm{Pe}_{i}}{d(d-1)}}, \\
& \gamma_{i}=\sqrt{\frac{d}{1+\frac{d(d-1)}{\mathrm{Pe}_{i}}},} \\
& \Lambda_{i}=\frac{1}{1+\frac{\mathrm{Pe}_{i}}{d(d-1)}} .
\end{aligned}
$$

Here, $n_{0}$ and $m_{x 0}$ are the number density and polar order at the step change in transport properties $(x=0)$. To determine the value of $n_{0}$, the particle conservation equation $\langle n\rangle=\int_{-L_{1}}^{L_{2}} n d x /\left(L_{1}+L_{2}\right)$ is used for given overall number density $\langle n\rangle$.

The analytic solution, however, is not enlightening nor easy to evaluate when the size of a domain is large. We instead have performed a singular perturbation analysis on the moment equations to obtain a more intuitive and simple analytical solution.

In the singular perturbation, we recognize that there are boundary layers with rapid variation in number density and polar order. They occur at the point of discontinuity in properties $(x=0)$ and at each wall. As long as the length $L_{i}$ is large compared to the screening length $\lambda_{i}^{-1}$, we can construct the full solution by piecing together the boundary layers. The singular perturbation solutions are valid when the dimension of the suspension is significantly larger than $D_{T} / U: L_{1} \gg D_{T 1} / U_{1}$ and $L_{2} \gg$ $D_{T 2} / U_{2}$.

The leading-order inner solutions within boundary layers near walls are given by Yan and Brady [26] as solutions for semiinfinite suspensions with one wall. Also the solution we have presented in Appendix A for the infinite suspension is the leadingorder inner solution within the boundary layer between two regions with different transport properties. The outer solutions far from boundary layers are homogeneous in the leading order: $n=n_{i}^{\text {bulk }}$ (const) and $\boldsymbol{m}=\mathbf{0}$. By matching inner and outer asymptotic solutions, we obtain the complete solution

$$
\begin{gathered}
\frac{n_{i}}{n_{i}^{\text {bulk }}}=1+\frac{1}{d(d-1)} \operatorname{Pe}_{i} e^{-\lambda_{i}\left(L_{i} \pm x\right)}+\frac{C_{i}\left(U_{1}-U_{2}\right)}{ \pm\left(\frac{U_{1}}{C_{1}}+\frac{U_{2}}{C_{2}}\right)-C_{i}\left(U_{1}-U_{2}\right)} e^{ \pm \lambda_{i} x}, \\
\frac{m_{i, x}}{n_{i}^{\text {bulk }}}=\frac{1}{\sqrt{d} C_{i}}\left[\mp \frac{1}{d(d-1)} \operatorname{Pe}_{i} e^{-\lambda_{i}\left(L_{i} \pm x\right)}+\frac{C_{i}\left(U_{1}-U_{2}\right)}{\left(\frac{U_{1}}{C_{1}}+\frac{U_{2}}{C_{2}}\right) \mp C_{i}\left(U_{1}-U_{2}\right)} e^{ \pm \lambda_{i} x}\right], \\
\frac{n_{1}^{\text {bulk }}}{n_{2}^{\text {bulk }}}=\frac{\left(\frac{U_{1}}{C_{1}}+\frac{U_{2}}{C_{2}}\right)-C_{1}\left(U_{1}-U_{2}\right)}{\left(\frac{U_{1}}{C_{1}}+\frac{U_{2}}{C_{2}}\right)+C_{2}\left(U_{1}-U_{2}\right)},
\end{gathered}
$$

where $\lambda_{i}$ and $C_{i}$ are the same as in (A5) and (A6). Note that $n_{1}^{\text {bulk }}$ and $n_{2}^{\text {bulk }}$ are the same if swim speeds in two regions are identical regardless of the other parameters-the number density is governed by swim speed. The bulk number densities $n_{1}^{\text {bulk }}$ and $n_{2}^{\text {bulk }}$ are determined by the conservation of particles:

$$
\int_{-L_{1}}^{0} n_{1} d z+\int_{0}^{L_{2}} n_{2} d z=\left(L_{1}+L_{2}\right)\langle n\rangle .
$$




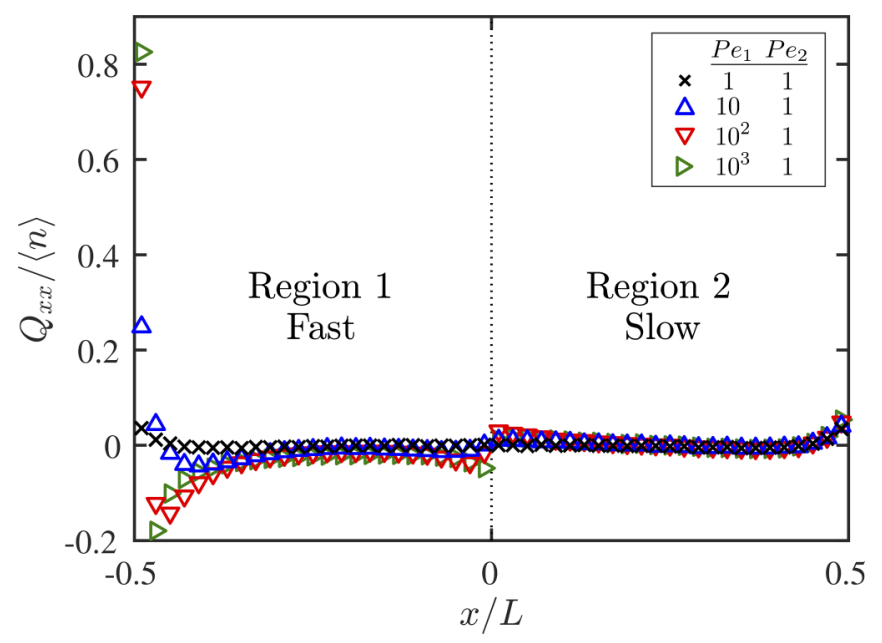

FIG. 8. The nematic order of ABPs (associated with Fig. 2) in a suspension confined between two walls scaled with the average number density $\langle n\rangle$. The swim speed of particles changes at $x=0$ and the position is scaled with the distance between the two walls $L$, which is 10 times longer than the microscopic length scale $\delta$. In all cases, the two regions have the same translational and rotational diffusivities and length $(L / 2)$, but the swim speeds differ: $\mathrm{Pe}_{1} / \mathrm{Pe}_{2}=$ $\left(U_{1} / U_{2}\right)^{2}$.

With BD simulations, we observe that the nematic order can become significant at very high activity. Figure 8 shows that boundary layers of the nematic order develop near the confining walls and near the step change in the swim speed of the particles. While the simple closure of $\boldsymbol{Q}=\mathbf{0}$ captures the essential physics, it can lose accuracy in the boundary layers for high activity.

Even though we do not present higher-order closures here, the shapes of boundary layers in the nematic order can be explained qualitatively. The nematic order drops sharply and becomes negative near the walls because most of the particles leave the walls orienting parallel to the walls. Near the step change in swim speed, two sharp peaks with different signs develop. The nematic order is coupled to the polar order by $\boldsymbol{Q} \sim U / D_{T} \nabla \boldsymbol{m}$ for high Pe. Since the polar order becomes a sharp peak near the step change, the nematic order becomes a derivative of the sharp peak, which resembles a shape of the derivative of a $\delta$-function.
Once the number density of suspended particles at a wall $n^{w}\left(\left.n\right|_{-L_{1}}\right.$ or $\left.\left.n\right|_{L_{2}}\right)$ is obtained, the pressure exerted by particles on the wall can be easily computed. The pressure exerted by particles on a wall is equivalent to the force density, or pressure, required to hold the wall at rest against the particles colliding into the wall. This pressure is simply given by $\Pi^{w}=n^{w} k_{B} T=n^{\text {bulk }} k_{B} T\left(1+D^{\text {swim }} / D_{T}\right)$ for a flat wall, where $D^{\text {swim }}=\ell^{2} /\left(2 \tau_{R}\right)$ is the energy scale of activity in two dimensions [26,34,35].

\section{APPENDIX D: ESTIMATION OF THE FLUID SPEED PUMPED BY MICROSWIMMERS WITH SPATIAL VARIATION IN SWIM SPEEDS}

We present steps of the calculation of the speed of the pumped fluid in a tube connecting two regions with different swim speeds, which is illustrated in Fig. 4. Since the difference in fluid pressure balances the difference in swim pressure, $\left|\Delta p_{f}^{w}\right|=\left|\Delta \Pi^{w}\right| \approx \zeta\langle n\rangle U_{h}|\Delta \ell| /[d(d-1)]$ for Pe $\gg 1$, where $U_{h}=2\left(U_{1}^{-1}+U_{2}^{-1}\right)^{-1}$ is the harmonic mean of the swim speeds in the two regions and $\Delta \ell$ is the difference in run lengths. For spherical active particles of radii $a$ and Stokes drag coefficient $\zeta=6 \pi \eta a,\left|\Delta p_{f}\right|=$ $(3 / 4) \eta \phi U_{h}|\Delta \ell| / a^{2}$, where $\phi$ is the averaged volume fraction of suspended particles and $\eta$ is the fluid viscosity. The pressure drop $\left|\Delta p_{m}\right|$ across a semipermeable membrane is estimated by Darcy's law: $\left|\Delta p_{m}\right|=\eta Q R_{m}$, where $Q$ is the volume flow-rate, $R_{m}=L_{m} /\left(\kappa A_{m}\right)$ is the resistance of the membrane, $\kappa$ is the permeability of the membrane, $A_{m}$ is the area, and $L_{m}$ is the membrane thickness. Assuming a cylindrical-shaped connecting tube and laminar flow, the pressure drop across the tube is $\left|\Delta p_{t}\right|=\eta Q R_{t}$, where $R_{t}=8 L_{t} /\left(\pi r_{t}^{4}\right)$ is the resistance of the tube and $L_{t}$ and $r_{t}$ are the length and radius of the tube. Finally, we obtain the average flow speed in the tube $u_{t}$ from $Q=$ $\pi r_{t}^{2} u_{t}=3 \phi U_{h}|\Delta \ell| /\left(8 R_{m}+4 R_{t}\right)$. If the tube is not too narrow $(\ll 1 \mathrm{~cm})$ or long $(\gg 1 \mathrm{~m})$, the resistance of membranes$L_{m} / \kappa \sim 10^{9} \mathrm{~m}^{-1}$ for Nuclepore membranes with a pore diameter of $1 \mu \mathrm{m}$ and even larger for smaller pores [36] — usually dominates, so we can approximate $u_{t} \approx 3 \phi U_{h}|\Delta \ell| /\left(8 \pi R_{m} r_{t}^{2}\right)$. For a suspension with volume fraction $\phi=0.2$ for $a=1 \mu \mathrm{m}$ and reorientation time $10 \mathrm{~s}$ with two regions with swim speeds 5 and $10 \mu \mathrm{m} / \mathrm{s}$ with $100-\mathrm{cm}^{2}$-wide membranes with $L_{m} / \kappa \sim 10^{9} \mathrm{~m}^{-1}$, the fluid speed in the tube of radius $1 \mathrm{~cm}$ is $0.1 \mu \mathrm{m} / \mathrm{s}$.
[1] C. Bechinger, R. Di Leonardo, H. Löwen, C. Reichhardt, G. Volpe, and G. Volpe, Rev. Mod. Phys. 88, 045006 (2016).

[2] Y. Fily and M. C. Marchetti, Phys. Rev. Lett. 108, 235702 (2012).

[3] I. Buttinoni, J. Bialké, F. Kümmel, H. Löwen, C. Bechinger, and T. Speck, Phys. Rev. Lett. 110, 238301 (2013).

[4] J. Palacci, S. Sacanna, A. P. Steinberg, D. J. Pine, and P. M. Chaikin, Science 339, 936 (2013).

[5] S. C. Takatori and J. F. Brady, Phys. Rev. E 91, 032117 (2015).
[6] A. P. Solon, J. Stenhammar, R. Wittkowski, M. Kardar, Y. Kafri, M. E. Cates, and J. Tailleur, Phys. Rev. Lett. 114, 198301 (2015).

[7] P. Digregorio, D. Levis, A. Suma, L. F. Cugliandolo, G. Gonnella, and I. Pagonabarraga, Phys. Rev. Lett. 121, 098003 (2018).

[8] P. Galajda, J. Keymer, P. Chaikin, and R. Austin, J. Bacteriol. 189, 8704 (2007).

[9] R. Di Giacomo, S. Krödel, B. Maresca, P. Benzoni, R. Rusconi, R. Stocker, and C. Daraio, Sci. Rep. 7, 45897 (2017). 
[10] H. M. López, J. Gachelin, C. Douarche, H. Auradou, and E. Clément, Phys. Rev. Lett. 115, 028301 (2015).

[11] D. Saintillan, Annu. Rev. Fluid Mech. 50, 563 (2018).

[12] M. Paoluzzi, R. Di Leonardo, and L. Angelani, Phys. Rev. Lett. 115, 188303 (2015).

[13] L. Angelani, A. Costanzo, and R. Di Leonardo, Europhys. Lett. 96, 68002 (2011).

[14] I. Buttinoni, G. Volpe, F. Kümmel, G. Volpe, and C. Bechinger, J. Phys. Condens. Matter 24, 284129 (2012).

[15] J. M. Walter, D. Greenfield, C. Bustamante, and J. Liphardt, Proc. Natl. Acad. Sci. (USA) 104, 2408 (2007).

[16] J. Hasnain, G. Menzl, S. Jungblut, and C. Dellago, Soft Matter 13, 930 (2017).

[17] J. Stenhammar, R. Wittkowski, D. Marenduzzo, and M. E. Cates, Sci. Adv. 2, e1501850 (2016).

[18] G. Frangipane, D. Dell'Arciprete, S. Petracchini, C. Maggi, F. Saglimbeni, S. Bianchi, G. Vizsnyiczai, M. L. Bernardini, and R. di Leonardo, eLife 7, e36608 (2018).

[19] J. Arlt, V. A. Martinez, A. Dawson, T. Pilizota, and W. C. Poon, Nat. Commun. 9, 768 (2018).

[20] M. J. Schnitzer, Phys. Rev. E 48, 2553 (1993).

[21] J. Tailleur and M. E. Cates, Phys. Rev. Lett. 100, 218103 (2008).

[22] M. E. Cates and J. Tailleur, Europhys. Lett. 101, 20010 (2013).

[23] M. E. Cates and J. Tailleur, Annu. Rev. Condens. Matter Phys. 6, 219 (2015).
[24] J. Arlt, V. A. Martinez, A. Dawson, T. Pilizota, and W. C. K. Poon, Nat. Commun. 10, 2321 (2019).

[25] When the temperature varies within a system, well-known thermal drifts [37] should be considered.

[26] W. Yan and J. F. Brady, J. Fluid Mech. 785, R1 (2015).

[27] For 1D processes one cannot set $d=1$ in Eq. (2) as the reorientation process is just changing direction \pm rather than an angular displacement as modeled in the Smoluchowski equation. The conclusion is the same, however, and the equivalent of $\boldsymbol{Q}=\mathbf{0}$ also holds.

[28] The nematic field associated with the steep change of $n$ and $\boldsymbol{m}$ needs to be included in the analysis as shown by Yan and Brady [26].

[29] A. K. Omar, Z.-G. Wang, and J. F. Brady, Phys. Rev. E 101, 012604 (2020).

[30] W. Yan and J. F. Brady, Soft Matter 11, 6235 (2015).

[31] S. C. Takatori and J. F. Brady, Curr. Opin. Colloid Interface Sci. 21, 24 (2016).

[32] A. K. Omar, Y. Wu, Z.-G. Wang, and J. F. Brady, ACS Nano 13, 560 (2019).

[33] D. R. Foss and J. F. Brady, J. Rheol. 44, 629 (2000).

[34] J. F. Brady, J. Chem. Phys. 98, 3335 (1993).

[35] T. M. Squires and J. F. Brady, Phys. Fluids 17, 073101 (2005).

[36] M. Cheryan, Ultrafiltration and Microfiltration Handbook (CRC, Boca Raton, FL, 1998).

[37] N. G. van Kampen, Z. Phys. B 68, 135 (1987). 